\title{
Deficiências e incapacidades em vítimas de acidentes de trânsito em Mato Grosso, Brasila
}

\section{Disabilities in victims of traffic accidents in Mato Grosso, Brazil}

\author{
Ligia Regina de Oliveira' (D), Flávia Guimarães Dias Duarte² (D) \\ 'Departamento de Saúde Coletiva, Instituto de Saúde Coletiva, Universidade Federal de Mato Grosso - Cuiabá (MT), Brasil. \\ ${ }^{2}$ Coordenadoria de Vigilância de Doenças e Agravos, Diretoria de Vigilância em Saúde, Secretaria Municipal de Saúde - Cuiabá \\ (MT), Brasil.
}

Como citar: Oliveira LR, Duarte FGD. Deficiências e incapacidades em vítimas de acidentes de trânsito em Mato Grosso, Brasil. Cad Saúde Colet, 2021;29(1):12-24. https://doi.org/10.1590/1414-462X202129010197

\section{Resumo}

Introdução: Acidentes de trânsito são importante problema de saúde pública, contudo pouco se conhece sobre as sequelas deles decorrentes. Objetivo: Analisar as deficiências e incapacidades em vítimas de acidentes de trânsito. Método: Estudo transversal com base em dados primários de uma amostra de vítimas de acidentes de trânsito não fatais atendidas em serviços selecionados de urgência e emergência de Mato Grosso em setembro de 2014. O instrumento de coleta foi baseado no Checklist da Classificação Internacional de Funcionalidade, Incapacidade e Saúde. Resultados: Foram avaliadas 228 vítimas de acidentes de trânsito, predominando jovens, sexo masculino, motociclistas. Deficiências de função do corpo mais comprometidas foram as sensoriais e dor $(49,3 \%)$ e neuromuscoloesqueléticas (45,8\%), enquanto as de estrutura do corpo relacionavam-se ao movimento (85,5\%); ambas apresentando gravidade moderada. Referente à limitação de atividade e restrição à participação, predominaram problemas relacionados à mobilidade (42,0\%), prevalecendo gravidade moderada (30,5\%). Dentre os fatores ambientais, destacaram-se como principais barreiras os serviços, sistemas e políticas $(66,3 \%)$ e, como facilitadores, o apoio e relacionamento com familiares e amigos (40,9\%). Conclusão: Aproximadamente metade das vítimas de acidentes de trânsito apresentou deficiências e incapacidades. O estudo preenche uma lacuna importante para a compreensão deste problema de saúde pública. Palavras-chave: acidentes de trânsito; Classificação Internacional de Funcionalidade, Incapacidade e Saúde (CIF); pessoas com deficiência; estatísticas de sequelas e incapacidade.

\section{Abstract}

Introduction: Traffic accidents are an important public health problem; however, little is known about their consequences. Objective: To analyze disabilities in traffic accident victims. Methods: Cross-sectional

\footnotetext{
Trabalho realizado no Instituto de Saúde Coletiva, Universidade Federal de Mato Grosso - Cuiabá (MT), Brasil. Correspondência: Ligia Regina de Oliveira. E-mail: Irdoliveira@gmail.com.

Fonte de Financiamento: Os dados utilizados neste estudo são provenientes da pesquisa "Violência e Acidentes: conhecendo os eventos não fatais e suas consequências" financiada pela Secretaria de Vigilância em Saúde do Ministério da Saúde a partir de sua aprovação no Chamamento Público no20/2013: Estudos e pesquisas aplicadas em Vigilância e Saúde.

Conflito de Interesses: nada a declarar.

Recebido em: Mai. 09, 2019. Aceito em: Jan. 13, 2020

a Este artigo compõe a dissertação intitulada 'Deficiências e incapacidades em vítimas de acidentes de trânsito atendidas em unidades de urgência e emergência em Mato Grosso' de Flávia Guimarães Dias Duarte, apresentada em 2016 junto ao Programa de Pós-Graduação em Saúde Coletiva do Instituto de Saúde Coletiva da Universidade Federal de Mato Grosso (UFMT) como requerimento parcial para a obtenção do título de Mestre em Saúde Coletiva. Este estudo integra o projeto de pesquisa "Violência e acidentes: conhecendo os eventos não fatais e suas consequências", financiado pela Secretaria de Vigilância em Saúde do Ministério da Saúde a partir de sua aprovação no Chamamento Público n²0/2013: Estudos e Pesquisas Aplicadas em Vigilância em Saúde. O projeto, executado no período de março de 2014 a setembro de 2015 , foi coordenado pelo Instituto de Saúde Coletiva da Universidade Federal de Mato Grosso e desenvolvido em parceria com a Secretaria Estadual de Saúde e 14 municípios sedes dos escritórios regionais de saúde do estado de Mato Grosso: Pontes e Lacerda, São Félix do Araguaia, Peixoto de Azevedo, Rondonópolis, Barra do Garças, Cáceres, Água Boa, Juína, Alta Floresta, Diamantino, Colíder, Juara, Tangará da Serra e Sinop.
} 
study, based on primary data from a sample of victims of non-fatal traffic accidents, attended at selected emergency services in Mato Grosso in September 2014. The collection instrument was based on the International Classification Checklist Functionality, Disability, and Health. Results: 228 victims of traffic accidents were evaluated, predominantly young men, motorcyclists. Body function deficiencies most affected were sensory and pain (49.3\%) and neuromusculoskeletal (45.8\%), while those of body structure were related to movement ( $85.5 \%)$; both presenting moderate severity. Regarding activity limitation and participation restriction, problems related to mobility predominated $(42.0 \%)$, with moderate severity (30.5\%). Among the environmental factors, services, systems, and policies stood out as barriers (66.3\%) and the support and relationship with family and friends as facilitators (40.9\%). Conclusion: Approximately half of the victims of traffic accidents had disabilities. The study fills an important gap for understanding this public health problem.

Keywords: traffic accidents; International Classification of Functioning, Disability, and Health (ICF); disabled people; sequel statistics and disability.

\section{INTRODUÇÃO}

Os acidentes de trânsito são um problema de grande magnitude e relevância para a saúde pública, estando entre as principais causas de morte, internações e atendimentos em unidades de urgência e emergência nos serviços de saúde. Estimativas da Organização Mundial de Saúde (OMS) indicam que a cada ano mais de 1,35 milhão de pessoas morre no mundo e dezenas de milhões ficam feridas em decorrência de acidentes de trânsito e, dessas, 10 milhões, com lesões incapacitantes e permanentes. ${ }^{1,2}$ Estima-se que, em 2020, os acidentes de trânsito serão a terceira causa de incapacidade, sendo a primeira entre a faixa etária produtiva. ${ }^{3}$

No Brasil, em 2017 ocorreram cerca de 36 mil mortes e 209 mil internações por acidentes de trânsito. ${ }^{4,5}$ Estudo realizado a partir de internações do Sistema Único de Saúde refere que cerca de $20 \%$ daqueles que evoluem para alta hospitalar apresentam algum tipo de sequela. ${ }^{6}$

A OMS instituiu, em 2001, a Classificação Internacional de Funcionalidade, Incapacidade e Saúde (CIF), traduzida e publicada no Brasil em 2003.7 A CIF define deficiência como problemas nas funções do corpo (funções fisiológicas dos sistemas orgânicos, incluindo as funções psicológicas) ou nas estruturas do corpo (partes anatômicas como órgãos, membros e seus componentes) enquanto a incapacidade é um termo que inclui deficiências, limitação de atividades ou restrição na participação. ${ }^{7}$

Baseada em um modelo biopsicossocial, a CIF constitui-se em um instrumento que serve de base conceitual para definir e medir as incapacidades. ${ }^{89}$ Neste modelo, a funcionalidade e a incapacidade são concebidas como uma interação dinâmica entre as condições de saúde (doença, trauma, lesões, distúrbios) e os fatores contextuais (pessoais e ambientais).,10,11 A $\mathrm{CIF}$ ainda é reconhecida como um avanço no que diz respeito às propostas de compreender as necessidades e as condições específicas das pessoas com deficiência assim como para a formulação de políticas para essa população. ${ }^{12}$

Apesar dos esforços na melhoria da informação sobre acidentes de trânsito, alguns autores declaram que ainda pouco se conhece sobre a magnitude dos eventos não fatais e, muito menos, sobre os tipos de sequelas e incapacidades deles decorrentes. ${ }^{9,13,14}$ MACEDO et al. (2014) afirmam que a utilização da CIF como instrumento na análise das consequências dos acidentes de trânsito por meio dos componentes biopsicossociais qualifica as informações sobre esses agravos, contribuindo para o cuidado e melhoria das condições de vida e saúde dos indivíduos. ${ }^{15}$

Reconhecendo a CIF como um instrumento que permite conhecer os aspectos relacionados à funcionalidade, capacidade e às situações de vida das pessoas, este estudo utilizou-se desta ferramenta com o objetivo de analisar as deficiências e incapacidades em vítimas de acidentes de trânsito atendidas em unidades de urgência e emergência de municípios mato-grossenses.

\section{MÉTODO}

Estudo do tipo transversal, com base em dados primários da pesquisa "Acidentes e violências: conhecendo os eventos não fatais e suas consequências".16 A partir do registro de 1.138 atendimentos de vítimas de acidentes de trânsito nas unidades de urgência e emergência 
dos municípios mato-grossenses de Barra do Garça, Rondonópolis, Sinop e Tangará da Serra, determinou-se a população deste estudo, constituída de 228 indivíduos.

O processo de amostragem foi do tipo sistemático, utilizando-se a equação recomendada por MARTINS (2001) para o cálculo amostral. Adotou-se na estimativa da amostra um erro de $4,8 \%$ indicando a distância entre a amostra e o parâmetro populacional, com proporção igual a 0,50; mantendo um intervalo no nível de confiança de $95,0 \%(Z=1,96) .{ }^{17}$

Foram incluídos na pesquisa indivíduos acima de 18 anos que concordaram em participar da pesquisa após leitura e assinatura do Termo de Consentimento Livre e Esclarecido.

Baseado no Checklist da $\mathrm{CIF}^{7}$ o instrumento de coleta dos dados foi criado pelo grupo de pesquisa ${ }^{16}$, sendo este testado previamente. O questionário semiestruturado constituía-se de variáveis relacionadas às características da vítima e do acidente. Além dessas, o instrumento de coleta apreendeu dados que permitiram, a partir do relato dos participantes, classificar as deficiências de funções e estruturas do corpo; as limitações de atividades e restrições à participação; assim como as barreiras e os facilitadores (fatores ambientais).? À esta classificação, foi acrescido um "qualificador" que especificava a extensão/gravidade do problema. Apoiandose na CIF, os qualificadores foram categorizados como: $1=$ leve, $2=$ moderada, $3=$ grave e 4 = completa. ${ }^{7}$ Para tanto, foi utilizada uma escala visual de graduação, na qual o indivíduo indicava a intensidade de cada problema.

A coleta de dados se deu em domicílio por pesquisadores capacitados. As entrevistas foram realizadas nos meses de novembro e dezembro de 2014, cerca de 60 dias após a data do atendimento das vítimas nas unidades de urgência e emergência. As questões referiam-se aos problemas enfrentados nos últimos 30 dias anteriores à entrevista.

Os dados foram digitados e processados com apoio do Microsoft Office Excel 2007 e sua análise contemplou a distribuição de frequência absoluta e relativa das variáveis categóricas.

O estudo foi aprovado pela Comissão de Ética em Pesquisa do Hospital Universitário Júlio Müller - UFMT (Processo № 093397/CEP-HUJM/2014).

\section{RESULTADOS}

Os resultados mostraram que, entre as 228 vítimas de acidente de trânsito atendidas nas unidades de urgência e emergência selecionadas, 136 (59,6\%) indivíduos foram identificados com deficiência de funções do corpo, cerca de 2,2 problemas por vítima, enquanto as deficiências nas estruturas do corpo foram observadas em 129 (56,6\%) vítimas (1,5/vítima). Referente à atividade e participação, foram identificados 100 (43,9\%) indivíduos com dificuldade em executar alguma atividade ou envolver-se nas situações sociais, resultando em 4,2 incapacidades por vítima. Em relação aos fatores ambientais, $37(17,2 \%)$ indivíduos identificaram barreiras, representando 1,5 barreiras por vítima e $75(32,9 \%)$ apresentaram situações que facilitaram seu desempenho (2,5/vítima).

Entre as características da população estudada, observou-se que a maioria foi do sexo masculino (62,3\%) e idade média de 31,9 anos, com intervalo entre 18 e 79 anos, sendo que mais da metade das vítimas tinha idade entre 20-39 anos (59,2\%). Prevaleceu a raça/cor parda/ preta $(65,8 \%)$ e ensino fundamental e médio, que representaram $43,0 \%$ (Tabela 1$)$.

O tipo de vítima mais frequente foi o condutor $(83,8 \%)$, sendo a motocicleta o meio de locomoção mais utilizado $(84,2 \%)$. As lesões que predominaram foram corte/laceração; entorse/luxação; contusão (78,5\%). As partes do corpo mais atingidas foram os membros inferiores $(39,1 \%)$ e cabeça/face $(34,2 \%)$ (Tabela 1$)$.

A utilização do capacete foi informada por $95,8 \%$ dos motociclistas, enquanto o uso de cinto de segurança foi revelado por $43,8 \%$ dos motoristas e passageiros de automóveis. 0 percentual da população estudada que referiu estar alcoolizada no momento do acidente foi de $8,5 \%$ e cerca de $1 / 3$ dos acidentes de trânsito estavam relacionados a acidente de trabalho.

A partir da classificação da funcionalidade e incapacidade, constatou-se que os problemas mais frequentes, nas vítimas de acidentes de trânsito estudadas, referiam-se à limitação de atividade e restrição à participação (419) e às deficiências de funções do corpo (205). 
Tabela 1. Características ( $n^{\circ}$ e \%) das vítimas e dos acidentes de trânsito segundo sexo. Barra do Garça, Rondonópolis, Sinop e Tangará da Serra, MT - 2014.

\begin{tabular}{|c|c|c|c|c|c|c|}
\hline \multirow{3}{*}{ Características } & \multicolumn{4}{|c|}{ SEXO } & \multirow{2}{*}{\multicolumn{2}{|c|}{ Total }} \\
\hline & \multicolumn{2}{|c|}{ Masculino } & \multicolumn{2}{|c|}{ Feminino } & & \\
\hline & $\mathbf{n}$ & $\%$ & $\mathbf{n}$ & $\%$ & $\mathbf{n}$ & $\%$ \\
\hline \multicolumn{7}{|l|}{ Faixa etária } \\
\hline $10-19$ & 30 & 21,1 & 8 & 9,3 & 38 & 16,7 \\
\hline $20-39$ & 78 & 54,9 & 57 & 66,3 & 135 & 59,2 \\
\hline $40-59$ & 25 & 17,6 & 18 & 20,9 & 43 & 18,9 \\
\hline 60 e mais & 9 & 6,3 & 3 & 3,5 & 12 & 5,3 \\
\hline \multicolumn{7}{|l|}{ Raça/cor* } \\
\hline Amarela & - & - & 2 & 2,3 & 2 & 0,9 \\
\hline Branca & 36 & 25,4 & 37 & 43,0 & 73 & 32,0 \\
\hline Indígena & 1 & 0,7 & 1 & 1,2 & 2 & 0,9 \\
\hline Parda/Preta & 105 & 73,9 & 45 & 52,4 & 150 & 65,8 \\
\hline \multicolumn{7}{|l|}{ Escolaridade* } \\
\hline Analfabeto & 3 & 2,2 & - & - & 3 & 1,4 \\
\hline Fundamental & 65 & 48,5 & 29 & 34,9 & 94 & 43,3 \\
\hline Ens. Médio & 54 & 40,3 & 40 & 48,2 & 94 & 43,3 \\
\hline Ens. Superior & 11 & 8,2 & 15 & 18,1 & 26 & 12,0 \\
\hline \multicolumn{7}{|l|}{ Tipo de vítima } \\
\hline Condutor & 132 & 93,0 & 59 & 68,6 & 191 & 83,8 \\
\hline Passageiro & 8 & 5,6 & 27 & 31,4 & 35 & 15,4 \\
\hline Pedestre & 2 & 1,4 & - & - & 2 & 0,9 \\
\hline \multicolumn{7}{|l|}{ Meio de locomoção } \\
\hline A pé & 2 & 1,4 & - & - & 2 & 0,9 \\
\hline Automóvel & 8 & 5,6 & 10 & 11,6 & 18 & 7,9 \\
\hline Bicicleta & 7 & 4,9 & 5 & 5,8 & 12 & 5,3 \\
\hline Motocicleta & 121 & 84,5 & 71 & 82,6 & 192 & 84,2 \\
\hline Outro** & 4 & 2,8 & - & - & 4 & 1,8 \\
\hline \multicolumn{7}{|l|}{ Natureza da lesão } \\
\hline Contusão & 15 & 10,6 & 15 & 17,4 & 30 & 13,2 \\
\hline Corte/laceração & 74 & 52,1 & 33 & 38,4 & 107 & 46,9 \\
\hline Entorse/luxação & 23 & 16,2 & 19 & 22,1 & 42 & 18,4 \\
\hline Fratura & 14 & 9,9 & 6 & 7,0 & 20 & 8,8 \\
\hline Politraumatismo & 5 & 3,5 & 3 & 3,5 & 8 & 3,5 \\
\hline
\end{tabular}

*Dados ignorados: 1 para Raça/cor e 11 para escolaridade. **Outro (ônibus/micro-ônibus, não se aplica e ignorado). 
Tabela 1. Continuação...

\begin{tabular}{|c|c|c|c|c|c|c|}
\hline \multirow{3}{*}{ Características } & \multicolumn{4}{|c|}{ SEXO } & \multirow{2}{*}{\multicolumn{2}{|c|}{ Total }} \\
\hline & \multicolumn{2}{|c|}{ Masculino } & \multicolumn{2}{|c|}{ Feminino } & & \\
\hline & $\mathbf{n}$ & $\%$ & $\mathbf{n}$ & $\%$ & $\mathbf{n}$ & $\%$ \\
\hline Outro & 6 & 4,2 & 10 & 11,6 & 16 & 7,0 \\
\hline Sem lesão física & 5 & 3,5 & - & - & 5 & 2,2 \\
\hline \multicolumn{7}{|c|}{ Parte do corpo atingida } \\
\hline Abdome/quadril & 3 & 2,2 & 4 & 4,7 & 7 & 3,1 \\
\hline Cabeça/face & 48 & 34,5 & 29 & 33,7 & 77 & 34,2 \\
\hline Coluna/medula & 2 & 1,4 & 1 & 1,2 & 3 & 1,3 \\
\hline Genitais/ânus & 1 & 0,7 & - & - & 1 & 0,4 \\
\hline Membros inferiores & 49 & 35,3 & 39 & 45,3 & 88 & 39,1 \\
\hline Membros superiores & 32 & 23,0 & 11 & 12,8 & 43 & 19,1 \\
\hline Tórax/dorso & 4 & 2,9 & 2 & 2,3 & 6 & 2,7 \\
\hline Não se aplica & 3 & 2,1 & - & - & 3 & 1,3 \\
\hline
\end{tabular}

*Dados ignorados: 1 para Raça/cor e 11 para escolaridade. **Outro (ônibus/micro-ônibus, não se aplica e ignorado).

As funções do corpo mais comprometidas foram as sensoriais e dor $(49,3 \%)$ e as neuromusculoesqueléticas (45,8\%) (Tabela 2). Em relação às primeiras, prevaleceram problemas relacionados a dor (85,5\%), podendo indicar sensação de dor generalizada ou localizada, em uma ou mais partes do corpo. As funções sensoriais envolveram visão, audição, paladar e outras, destas, o maior acometimento esteve vinculado à temperatura e a outros estímulos $(9,0 \%)$. Em relação às deficiências neuromuscoloesqueléticas, as mais comprometidas foram a força muscular (32,1\%), relacionada à força gerada pela contração de um ou grupo de músculo, e a mobilidade das articulações $(30,4 \%)$, que se refere à amplitude e facilidade de movimento de uma articulação (Tabela 2).

Quanto às deficiências de estrutura do corpo (Tabela 2), 85,5\% relacionaram-se ao movimento, com as maiores alterações na estrutura das extremidades inferiores $(43,6 \%)$ e superiores $(20,1 \%)$, seguidas pelas estruturas do ombro $(14,6 \%)$, pele e estruturas $(10,9 \%)$, cabeça e pescoço $(10,4 \%)$ e tronco $(9,1 \%)$.

Entre os problemas relativos à execução de atividades ou participação social (Tabela 3), destacou-se a mobilidade $(174 ; 42,0 \%)$, limitando atividades como as de levantar e carregar objetos $(17,8 \%)$, andar $(16,7 \%)$ ou dirigir $(15,5 \%)$. Na sequência, sobressaíram os cuidados pessoais $(57 ; 13,6 \%)$, como lavar-se $(34,5 \%)$ e vestir-se $(31,0 \%)$.

Ainda foram identificados problemas em relação às tarefas e demandas gerais, vida doméstica e vida comunitária, representando o mesmo percentual de comprometimento $(11,0 \%)$. Alusivo às tarefas e demandas gerais, sobressaiu a realização da rotina diária $(41,3 \%)$ referente à vida comunitária, a recreação e o lazer foram os problemas mais prevalentes $(76,1 \%)$ (Tabela 3).

Dentre os fatores ambientais, os serviços, sistemas e políticas ofereceram as maiores barreiras $(66,3 \%)$ às vítimas de acidente de trânsito. Entre esses, destacaram-se os relacionados à saúde $(40,5 \%)$ e ao trabalho e emprego $(32,4 \%)$ (Tabela 4). Observou-se ainda em menor proporção que os produtos e tecnologias (20,0\%), atitudes (5,5\%), ambiente natural e mudanças ambientais feitas pelo ser humano $(3,6 \%)$ e apoio e relacionamentos $(3,6 \%)$ foram outras barreiras referidas pela população estudada. 
Tabela 2. Deficiências ( $n^{\circ}$ e \%) em vítimas de acidentes de trânsito segundo capítulos e categorias da CIF*. Barra do Garça, Rondonópolis, Sinop e Tangará da Serra - Mato Grosso, 2014.

\begin{tabular}{|c|c|c|}
\hline Capítulos/Categorias & $\mathbf{n}$ & $\%$ \\
\hline Funções do corpo & 295 & 100,0 \\
\hline Sensoriais e dor (b210-b299) & 145 & 49,2 \\
\hline Temperatura e outros estímulos (b270) & 13 & 9,0 \\
\hline Sensação de dor (b280) & 124 & 85,5 \\
\hline Outras $^{1}$ & 8 & 5,5 \\
\hline Neuromusculoesqueléticas (b710-b799) & 135 & 45,7 \\
\hline Mobilidade das articulações (b710) & 41 & 30,4 \\
\hline Força muscular (b730) & 43 & 31,9 \\
\hline Resistência muscular (b740) & 14 & 10,4 \\
\hline Padrão de marcha (b770) & 19 & 14,1 \\
\hline Outros $^{2}$ & 18 & 13,3 \\
\hline Demais funções ${ }^{3}$ & 15 & 5,1 \\
\hline Estruturas do corpo & 193 & 100,0 \\
\hline Relacionadas ao movimento (s710-s799) & 165 & 85,5 \\
\hline Região da cabeça e do pescoço (s710) & 17 & 10,4 \\
\hline Ombro (s720) & 24 & 14,6 \\
\hline Extremidade Superior (s730) & 33 & 20,1 \\
\hline Extremidade inferior (s750) & 72 & 43,6 \\
\hline Tronco (s760) & 15 & 9,1 \\
\hline Outras ${ }^{4}$ & 4 & 1,2 \\
\hline Pele e estruturas relacionadas (s810-s899) & 21 & 11,4 \\
\hline Áreas da pele (s810) & 21 & 100,0 \\
\hline Demais estruturas $^{5}$ & 7 & 3,1 \\
\hline
\end{tabular}

*Classificação Internacional de Funcionalidade, Incapacidade e Saúde. ${ }^{1}$ Audição e função vestibular (1); Proprioceptiva (7). ${ }^{2}$ Estabilidade das articulações (5); Tônus muscular (13). ${ }^{3}$ Mentais (11); Voz e fala (1); Sistema cardiovascular e respiratório (1); Pele e estruturas relacionadas (2). ${ }^{4}$ Região pélvica (2); Outras específicas (2). ${ }^{5}$ Sistema nervoso (2); Voz e fala (5).

Entre os fatores que facilitaram a vida das vítimas, prevaleceram o apoio e relacionamentos $(40,9 \%)$ com familiares, amigos, colegas, vizinhos e outros, e, dentre esses, com destaque para a família imediata $(71,4 \%)$ e amigos $(14,5 \%)$. Produtos e tecnologias $(30,9 \%)$ também se destacaram, sendo os produtos ou substâncias para o consumo pessoal os mais referidos $(67,2 \%)$. Em menor proporção, foram mencionados como facilitadores, os serviços, sistemas e políticas $(16,4 \%)$, com destaque para os serviços, sistemas e políticas de saúde $(80,0 \%)$ e, entre as atitudes $(11,7 \%)$, as relacionadas à família foram as mais mencionadas $(54,0 \%)$.

A análise da extensão das deficiências de função e de estrutura do corpo mostrou que essas apresentaram principalmente gravidade moderada, respectivamente $34,5 \%$ e 31,3\%. Tal como para as deficiências de função e estrutura do corpo, as limitações de atividade e restrição à participação foram predominantemente de magnitude moderada (30,5\%). Tanto as barreiras $(52,8 \%)$ como os facilitadores (64,5\%) foram majoritariamente de magnitude completa (Tabela 5). 
Tabela 3. Limitação de atividades e restrição à participação ( $\left.n^{\circ} \mathrm{e} \%\right)$ em vítimas de acidentes de trânsito segundo capítulos e categorias da CIF*. Barra do Garça, Rondonópolis, Sinop e Tangará da Serra - Mato Grosso, 2014.

\begin{tabular}{|c|c|c|}
\hline Capítulos/Categorias & $\mathbf{n}$ & $\%$ \\
\hline Atividades e participação & 418 & 100,0 \\
\hline Tarefas e demandas gerais (d210-d299) & 46 & 11,0 \\
\hline Realizar uma única tarefa (d210) & 12 & 26,1 \\
\hline Realizar tarefas múltiplas (d220) & 14 & 30,4 \\
\hline Realizar a rotina diária (d230) & 19 & 41,3 \\
\hline Tarefas e demandas gerais, outras (d298) & 1 & 2,2 \\
\hline Mobilidade (d410-d499) & 174 & 41,6 \\
\hline Mudar a posição básica do corpo (d410) & 20 & 11,5 \\
\hline Mudar a posição do corpo (d415) & 19 & 10,9 \\
\hline Levantar e carregar objetos (d430) & 31 & 17,8 \\
\hline Andar (d450) & 29 & 16,7 \\
\hline Dirigir (d475) & 27 & 15,5 \\
\hline Outros $^{1}$ & 48 & 27,6 \\
\hline Cuidado pessoal (d510-d599) & 57 & 13,7 \\
\hline Lavar-se (d510) & 20 & 35,1 \\
\hline Vestir-se (d540) & 18 & 31,6 \\
\hline Outros $^{2}$ & 19 & 33,3 \\
\hline Vida doméstica (d610-d699) & 45 & 11,0 \\
\hline Preparação de refeições (d630) & 12 & 26,7 \\
\hline Realização de tarefas domésticas (d640) & 24 & 53,3 \\
\hline Outros $^{3}$ & 9 & 20,0 \\
\hline Vida comunitária (d910-d999) & 46 & 11,0 \\
\hline Vida comunitária (d910) & 10 & 21,7 \\
\hline Recreação e lazer (d920) & 35 & 76,1 \\
\hline Religião e espiritualidade (d930) & 1 & 2,2 \\
\hline Demais atividades ${ }^{4}$ & 50 & 11,7 \\
\hline
\end{tabular}

*Classificação Internacional de Funcionalidade, Incapacidade e Saúde. ${ }^{1}$ Transferir a própria posição (13); Mover objetos com as extremidades inferiores (2); Uso fino da mão (5); Deslocar-se (12); Deslocar-se por diferentes locais (1); Deslocar-se utilizando algum tipo de equipamento (7); Utilização de transporte (8). ${ }^{2}$ Cuidado das partes do corpo (5); Cuidados relacionados aos processos de excreção (5); Comer (3); Beber (1). ${ }^{3}$ Aquisição de bens e serviços (6); Cuidar dos objetos da casa (2); Ajudar os outros (1). ${ }^{4}$ Relações e interações interpessoais (16); Áreas principais da vida (34).

\section{DISCUSSÃO}

Este estudo descreveu a funcionalidade e a gravidade/extensão das deficiências e incapacidades em vítimas não fatais de acidente de trânsito atendidas nas unidades de urgência e emergência dos municípios selecionados de Mato Grosso, Brasil. Pôde-se ainda propiciar maior visibilidade da CIF como ferramenta para classificar as diversas condições de saúde do 
Tabela 4. Classificação dos fatores ambientais - barreiras e facilitadores ( $n^{\circ}$ e \%) - em vítimas de acidente de trânsito segundo capítulos e categorias da CIF*. Barra do Garça, Rondonópolis, Sinop e Tangará da Serra - Mato Grosso, 2014.

\begin{tabular}{|c|c|c|}
\hline Capítulos/Categorias & $\mathbf{N}$ & $\%$ \\
\hline Fatores ambientais - barreiras & 55 & 100,0 \\
\hline Produtos e tecnologias (e110-e199) & 11 & 20,0 \\
\hline Ambiente natural e mudanças ambientais (e210-e299) & 2 & 3,6 \\
\hline Apoio e relacionamentos (e310-e399) & 2 & 3,6 \\
\hline Atitudes (e410-e499) & 3 & 5,5 \\
\hline Serviços, sistemas e políticas (e510-e599) & 37 & 66,3 \\
\hline Serviços, sist. e políticas da previdência social (e570) & 5 & 13,5 \\
\hline Serviços, sistemas e políticas de saúde (e580) & 15 & 40,6 \\
\hline Serviços, sist. e políticas de trabalho e emprego (e590) & 12 & 32,4 \\
\hline Outros $^{1}$ & 5 & 13,5 \\
\hline Fatores ambientais - facilitadores & 188 & 100,0 \\
\hline Produtos e tecnologias (e110-e199) & 58 & 30,9 \\
\hline Produtos ou substâncias para consumo pessoal (e110) & 38 & 65,5 \\
\hline Produtos e tecnologia uso pessoal na vida diária (e115) & 9 & 15,5 \\
\hline $\begin{array}{l}\text { Produtos e tecnologia para mobilidade e transporte pessoal em ambientes internos } \\
\text { e externos (e120) }\end{array}$ & 9 & 15,5 \\
\hline Outros $^{2}$ & 2 & 3,5 \\
\hline Apoio e relacionamentos (e310-e399) & 77 & 40,9 \\
\hline Família imediata (e310) & 55 & 71,4 \\
\hline Amigos (e320) & 11 & 14,3 \\
\hline Outros $^{3}$ & 11 & 14,3 \\
\hline Atitudes (e410-e499) & 22 & 11,7 \\
\hline Atitudes individuais de membros da família imediata (e410) & 12 & 54,6 \\
\hline Atitudes individuais dos profissionais de saúde (e450) & 5 & 22,7 \\
\hline Outros $^{4}$ & 5 & 22,7 \\
\hline Serviços, sistemas e políticas (e510-e599) & 31 & 16,5 \\
\hline Serviços, sistemas e políticas de transporte (e540) & 2 & 6,5 \\
\hline Serviços, sistemas e políticas de saúde (e580) & 24 & 77,4 \\
\hline Serviços, sist. e políticas de trabalho e emprego (e590) & 5 & 16,1 \\
\hline
\end{tabular}

*Classificação Internacional de Funcionalidade, Incapacidade e Saúde. ' Serviços, sistemas e políticas dos serviços públicos (1); Serviços, sistemas e políticas de comunicação (1); Serviços, sistemas e políticas de transporte (3). ${ }^{2}$ Produtos e tecnologia relacionados ao uso e à exploração do solo (1); Bens (1). ${ }^{3}$ Família ampliada (1); Conhecidos, companheiros, colegas, vizinhos e membros da comunidade (4); Pessoas em posição de autoridade (4); Profissionais de saúde (2). ${ }^{4}$ Atitudes individuais dos amigos (2); Atitudes individuais de conhecidos, companheiros, colegas, vizinhos e membros da comunidade (3). 
Tabela 5. Extensão das deficiências e incapacidades (\%) em vítimas de acidente de trânsito segundo componentes da CIF*. Barra do Garça, Rondonópolis, Sinop e Tangará da Serra - Mato Grosso, 2014.

\begin{tabular}{|c|c|c|c|c|}
\hline \multirow{2}{*}{ Componente } & \multicolumn{4}{|c|}{ Extensão } \\
\hline & Leve $^{1}$ & Moderada $^{2}$ & Grave $^{3}$ & Completa $^{4}$ \\
\hline Funções do corpo & 32,8 & 34,5 & 23,9 & 8,8 \\
\hline Sensorial e dor & 31,0 & 40,7 & 21,4 & 6,9 \\
\hline Neuromusculoesqueléticas & 37,3 & 26,9 & 24,6 & 11,2 \\
\hline Estruturas do corpo & 29,7 & 31,3 & 23,4 & 15,6 \\
\hline Movimento & 28,5 & 29,0 & 25,5 & 17,0 \\
\hline Atividades e participação & 28,2 & 30,5 & 18,1 & 23,2 \\
\hline Mobilidade & 27,6 & 28,7 & 23,6 & 20,1 \\
\hline Cuidado pessoal & 32,8 & 37,9 & 15,5 & 13,8 \\
\hline Fatores ambientais - barreiras & 14,5 & 14,5 & 18,2 & 52,8 \\
\hline Serviços, sistemas e políticas & 13,5 & 10,8 & 21,6 & 54,1 \\
\hline Fatores ambientais - facilitadores & 4,4 & 10,4 & 20,7 & 64,5 \\
\hline Apoio e relacionamento & 2,6 & 3,9 & 13,2 & 80,3 \\
\hline Produtos e tecnologias & 7,0 & 14,0 & 22,8 & 56,2 \\
\hline
\end{tabular}

*Classificação Internacional de Funcionalidade, Incapacidade e Saúde. ${ }^{1}$ Problema presente em menos que $25 \%$ do tempo, intensidade tolerável e ocorre raramente nos últimos 30 dias. ${ }^{2}$ Problema presente em menos que $50 \%$ do tempo, intensidade que interfere na vida e ocorre ocasionalmente nos últimos 30 dias. ${ }^{3}$ Problema presente em menos que $25 \%$ do tempo, intensidade tolerável e ocorre raramente nos últimos 30 dias. ${ }^{4}$ Problema presente em mais que $95 \%$ do tempo, intensidade que prejudica totalmente a vida e ocorre todos os dias nos últimos 30 dias.

indivíduo que experimenta qualquer deficiência ou incapacidade, transitória ou permanente, decorrente dos acidentes de trânsito.

Referente às características das vítimas não fatais de acidentes de trânsito atendidas em unidades de urgência e emergência, foram encontrados resultados semelhantes em outras pesquisas, apontando para maior vulnerabilidade do adulto jovem do sexo masculino que conduz motocicleta. ${ }^{18,19,20,21}$

Os tipos de lesões que prevaleceram sugerem o caráter mais leve das lesões em vítimas não fatais atendidas em unidades de urgência e emergência apontado por outros estudos. ${ }^{18,19,20,22}$

O predomínio de motociclistas, revelado por este estudo, também referenciado em estudos de morbimortalidade e de demanda ${ }^{22,23,24}$, pode ser atribuído às facilidades financeiras para adquirir motocicletas e às diversas opções do mercado de trabalho como motoboys e mototaxistas. ${ }^{25,26}$

A maior prevalência de membros inferiores e superiores pode ser explicada pelo alto índice de motociclistas no estudo e de utilização do capacete por esses, protegendo cabeça/face, igualmente evidenciado neste estudo. Por outro lado, o estudo também mostrou o baixo percentual do uso de cinto de segurança, sugerindo a desatenção dos motoristas e passageiros de automóveis quanto ao uso de equipamentos de segurança e ao cumprimento da lei, o que pode ter influenciado a proporção de lesões de cabeça/face. As legislações e normas para a utilização do cinto de segurança e capacete estão vigentes há vários anos, com penalidade para os infratores, mesmo assim são notórios o desrespeito e a exposição ao risco, o que aumenta a gravidade e letalidade das lesões. ${ }^{26,27,28}$

Cerca de $40 \%$ da população mundial com mais de 15 anos consome alguma forma de álcool. ${ }^{29}$ Além do mais, o álcool figura nas pesquisas como um dos fatores de risco para as 
mortes prematuras e incapacidades. ${ }^{30,31}$ Assim como em outros estudos ${ }^{15,22,24,31,32,33}$, parte da população investigada referiu estar alcoolizada no momento do acidente, apontando para o não cumprimento da legislação vigente no país e a naturalização do uso de álcool em nossa sociedade. Resultados da Pesquisa Nacional de Saúde, realizada em 2013 no Brasil, indicam que Mato Grosso é o segundo estado com maior prevalência de acidente de trânsito dentre os indivíduos que consomem álcool abusivamente. ${ }^{34}$

Este estudo revelou ainda que parte importante dos acidentes de trânsito estava relacionada à acidente de trabalho. Tal situação demanda atenção dos órgãos do trabalho, além dos de saúde, para ações de promoção, prevenção e controle, visto que muitas vítimas eram motociclistas com a função de motoboy, que, em geral, muitas vezes recebem por entrega, o que pode aumentar o risco, pela velocidade e exposição para garantir uma renda maior. ${ }^{25,26}$

As deficiências de função do corpo que prevaleceram nas vítimas não fatais de acidentes de trânsito foram a dor e neuromusculoesqueléticas. Outros estudos que utilizaram a CIF para classificação das alterações funcionais do corpo também apontam essas como as mais frequentes. ${ }^{31,35} \mathrm{O}$ trauma decorrente de acidentes de trânsito reflete em inúmeras modificações no conjunto de tecidos, podendo afetar pele, músculos, ossos, nervos entre outros, retratando os resultados encontrados neste estudo.

O comprometimento dessas funções, assim como as deficiências de estruturas do corpo observadas, pode ter contribuído para a prevalência das incapacidades (limitações de atividades e restrições à participação) identificadas neste estudo. Tais resultados corroboram estudo em vítimas de acidentes de trânsito em reabilitação. ${ }^{15,36}$ Também SABINO et al. (2008) e LUCENA et al. (2011) apresentaram achados semelhantes quando descreveram, em seus estudos de base populacional, que as categorias mais comprometidas foram as relacionadas à mobilidade dos sujeitos e cuidados pessoais. ${ }^{37,38}$

No modelo da CIF, a funcionalidade e a incapacidade são concebidas como uma interação dinâmica entre as condições de saúde e os fatores contextuais que interagem com as funções e estrutura do corpo, além das atividades e participação. 9,39,40,41 MORETTIN et al. (2008) discutiram a importância dos fatores ambientais pelo reconhecimento do contexto individual, permitindo avaliar as distintas experiências no processo saúde-doença, os diferentes resultados obtidos com a mesma terapêutica e as diversas reações ao vivenciar a incapacidade. ${ }^{42}$

Neste estudo, os fatores ambientais que atuaram como facilitadores mostraram-se mais representativos que as barreiras no desempenho dos indivíduos estudados. Essas últimas foram representadas principalmente pelos serviços, sistemas e políticas responsáveis por prover, controlar e normatizar os vários setores da sociedade. O fato de as barreiras referirem especialmente aspectos relacionados aos serviços de saúde, implica, de alguma forma, a necessidade de se redefinir as estratégias de atuação do sistema em relação às vítimas de acidente de trânsito.

O apoio e relacionamento com familiares, amigos, colegas, vizinhos e outros mostraram-se importante fator facilitador para as vítimas não fatais de acidentes de trânsito. RIBERTO et al. (2011) identificaram os mesmos agrupamentos como facilitadores no estudo realizado para validação do core set da CIF para lombalgia. ${ }^{14}$

Os fatores ambientais influenciam o processo de funcionalidade e incapacidade dos indivíduos pela interação com os demais componentes da CIF (funções corporais, estruturas corporais, fatores pessoais, atividades e participação), daí a importância de analisar os impactos à saúde a partir deles, haja vista sua vinculação com o desempenho humano, a participação social e as condições de vida e saúde da população. ${ }^{14,39,41,42,43}$

Em estudo de revisão sistemática da literatura, ATHAYDE et al. (2017) concluem que o ambiente físico e social pode ter sua interferência atribuída a diferentes condições clínicas. ${ }^{44}$ Os mesmos autores ainda afirmam ser relevante que o componente ambiental seja compreendido e avaliado integralmente.

A análise da extensão ou gravidade das deficiências e incapacidades das vítimas de acidente de trânsito revelou que tanto as deficiências (função e estrutura do corpo) como as limitações de atividade e restrição à participação foram predominantemente de extensão moderada, significando que o problema está presente entre $25 \%$ e $49 \%$ do tempo, com 
intensidade que interfere na vida diária da pessoa e que ocorre frequentemente. ${ }^{7}$ Constatou-se, mesmo sendo a maioria das lesões classificadas como leves, um comprometimento importante para as vítimas. Embora não tenham prevalecido fraturas, amputações, alterações no sistema nervoso central, a dor e as alterações musculoesqueléticas estão presentes no cotidiano das vítimas, sugerindo comprometimento no desempenho das atividades da vida diária.

O fato de grande parte dos problemas referentes aos fatores ambientais apresentarem extensão grave (problema presente entre 50\% e 95\% do tempo, com intensidade que prejudica/ rompe parcialmente a vida diária da pessoa) ou completa (problema se apresenta entre $96 \%$ e $100 \%$ do tempo e ocorre todos os dias com intensidade que prejudica/rompe totalmente a vida diária da pessoa) ${ }^{7}$ sugere que os fatores ambientais, sejam facilitadores sejam barreiras, interferem expressivamente no cotidiano da vida de vítimas não fatais de acidentes de trânsito. Neste sentido, este estudo ratifica os princípios da CIF que assinalam o importante papel dos fatores ambientais na expressão da funcionalidade e incapacidade humana.

O uso da CIF em pesquisas ainda é bastante reduzido, o que limitou não somente a comparação dos resultados apontados como também a identificação de métodos apropriados para consolidação e análise dos dados. Pelo fato de não ter sido realizada análise inferencial, os resultados deste estudo não podem ser generalizados e, portanto, devem ser apreendidos com cautela.

Mesmo diante destas limitações, ressalta-se a relevância dos achados, considerando que são raros os estudos que abordam a funcionalidade em vítimas de acidentes de trânsito.

Este estudo produziu informações relevantes sobre um dos principais problemas de saúde da atualidade na medida em que apresentou a prevalência de deficiências e incapacidades em vítimas de acidentes de trânsito, mostrando a importância da abordagem multidimensional, transcendendo o olhar biomédico. Estudos desta natureza poderão contribuir para o planejamento de ações e serviços de saúde, possibilitando a melhoria da qualidade de vida e reintegração social desses indivíduos.

Foi evidenciado que a CIF é uma ferramenta útil para compreender a funcionalidade humana a partir da integração dos aspectos biopsicossociais, portanto seu uso em pesquisas deve ser estimulado. A informação sobre o diagnóstico acrescido da funcionalidade fornece um quadro mais amplo sobre a saúde dos indivíduos ou das populações.

\section{REFERÊNCIAS}

1. Organização Pan-Americana da Saúde. Informe sobre segurança no trânsito na Região das Américas. Washington. OPAS; 2015.

2. World Health Organization. Global status report on road safety. Genebra: WHO; 2018.

3. Ameratunga SN, Norton RN, Bennett DA, Jackson RT. Risk of disability due to car crashes: a review of the literature and methodological issues. Injury. 2004;35(11):1116-27. http://dx.doi.org/10.1016/j. injury.2003.12.016. PMid:15488502.

4. Brasil. Ministério da Saúde. Secretaria de Vigilância da Saúde. Departamento de Análise de Situação de Saúde. [Internet]. 2019a [citado em 2019 mai 30]. Disponível em: http://tabnet.datasus.gov.br/cgi/tabcgi. exe?sim/cnv/ext10br.def.

5. Brasil. Ministério da Saúde. Secretaria de Vigilância da Saúde. Departamento de Análise de Situação de Saúde [Internet]. 2019b. [citado em 2019 jun 18]. Disponível em: http://tabnet.datasus.gov.br/cgi/tabcgi. exe?sih/cnv/fruf.def.

6. Di Nubila HBV. Uma introdução à CIF - Classificação Internacional de Funcionalidade, Incapacidade e Saúde. Rev Bras Saúde Ocup. 2010;35(121):122-3. http://dx.doi.org/10.1590/\$0303-76572010000100013.

7. Organização Mundial de Saúde. CIF: Classificação Internacional de Funcionalidade, Incapacidade e Saúde. São Paulo: Editora da Universidade de São Paulo; 2015. (Centro Colaborador da OMS para a Família de Classificações Internacionais, org.; coordenação da tradução Cássia Maria Buchalla, $1^{\text {a }}$ ed., p. 334).

8. Venturini DA, Decésaro MN, Marcon SS. Alterações e expectativas vivenciadas pelos indivíduos com lesão raquimedular e suas famílias. Rev Esc Enferm USP. 2007;41(4):589-96.

9. Brasil. Secretaria de Direitos Humanos da Presidência da República. Secretaria Nacional de Promoção dos Direitos da Pessoa com Deficiência. Coordenação-Geral do Sistema de Informações sobre a Pessoa com Deficiência. Cartilha do Censo 2010 - Pessoas com Deficiência. Brasília: SDH PR/SNPD; 2012. 32 p. 
10. Franzoi AC, Xerez DR, Blanco M, Amaral T, Costa AJ, Khan P. et al. Etapas da elaboração do instrumento de Classificação do Grau de Funcionalidade de Pessoas com Deficiência para Cidadãos Brasileiros: Índice de Funcionalidade Brasileiro-IF-BR. Acta Fisiátrica. 2013;20(3):164-178.

11. Farias N, Buchalla CMA. Classificação internacional de funcionalidade, incapacidade e saúde da organização mundial da saúde: conceitos, usos e perspectivas. Rev Bras Epidemiol. 2005;8(2):187-93. http://dx.doi. org/10.1590/S1415-790X2005000200011.

12. Lemos CAG, Jorge MT, Ribeiro LA. Perfil de vítimas e tratamento de lesões por causas externas segundo atendimento pelo Centro de Reabilitação Municipal de Uberlândia, MG - Causas externas e fisioterapia. Rev Bras Epidemiol. 2013;16(2):482-92. http://dx.doi.org/10.1590/S1415-790X2013000200022. PMid:24142018.

13. Buchalla CM. A classificação internacional de funcionalidade, incapacidade e saúde. Acta Fisiátrica. 2003;10(1):29-31.

14. Riberto M. Core sets da classificação internacional de funcionalidade, incapacidade e saúde. Rev Bras Enferm. 2011;64(5):938-46. http://dx.doi.org/10.1590/S0034-71672011000500021. PMid:22460498.

15. Macedo APFS, Oliveira LR, Buchalla CM, Scatena JHG. Características e deficiências físicas de vítimas de acidentes de trânsito atendidas no serviço de referência para reabilitação do estado de Mato Grosso, Brasil, 2010. Revista Espaço para a Saúde. 2014;15(4):21-33. http://dx.doi.org/10.22421/15177130.2014v15n4p22.

16. Oliveira, LR. Acidentes e violência: conhecendo os eventos não fatais e suas consequências. Estudos e Pesquisas Aplicadas em Vigilância em Saúde Ministério da Saúde. Secretaria de Vigilância em Saúde. Chamamento Público n²0/2013. Cuiabá, MT.

17. Martins GA. Estatística geral e aplicada. São Paulo: Atlas; 2001.

18. Marchese VS, Scatena JHG, Ignotti E. Caracterização das vítimas de acidentes e violências atendidas em serviços de emergência. Município de Alta Floresta, MT (Brasil). Rev Bras Epidemiol. 2008;11(4):648-59. http://dx.doi.org/10.1590/S1415-790X2008000400012.

19. Oliveira LR, Mello Jorge MHP. Acidentes de transporte em Cuiabá, Mato Grosso: morbi-mortalidade. Revista ABRAMET. 2008;26:36-45.

20. Soares BAC, Scatena JHG, Galvão ND. Acidentes e violências na Grande Cuiabá: o que retrata a demanda dos serviços de emergência. Epidemiol Serv Saude. 2009;18(3):265-76.

21. Mascarenhas MDM, Silva MMA, Malta DC, Moura L, Gawryszewski VP, Costa VC, et al. Atendimentos de emergência por acidentes na Rede de Vigilância de Violências e Acidentes - Brasil. 2006. Cien Saude Colet. 2009;14(5):1657-68. http://dx.doi.org/10.1590/S1413-81232009000500007. PMid:19851578.

22. Malta DC, Bernal RTI, Mascarenhas MDM, Monteiro RA, Sá NNB, Andrade SSCA, et al. Atendimentos por acidentes de transporte em serviços públicos de emergência em 23 capitais e no Distrito Federal - Brasil, 2009. Epidemiol Serv Saude. 2012;21(1):31-42. http://dx.doi.org/10.5123/S1679-49742012000100004.

23. Brasil. Ministério da Saúde. Secretaria de Vigilância em Saúde. Departamento de Vigilância de Doenças e Agravos Não Transmissíveis e Promoção da Saúde. Viva: Vigilância de Violências e Acidentes: 2013 e 2014 [Internet]. Brasília, 2017. 218 p. [citado em 2019 Maio 09]. Disponível em: http://bvsms.saude.gov. br/bvs/publicacoes/viva_2013_2014.pdf

24. Golias ARC, Caetano R. Acidentes entre motocicletas: análise dos casos ocorridos no estado do Paraná entre julho de 2010 e junho de 2011. Cien Saude Colet. 2012;18(5):1235-46. http://dx.doi.org/10.1590/ S1413-81232013000500008. PMid:23670451.

25. Bacchieri G, Barros AJD. Acidentes de trânsito no Brasil de 1998 a 2010: muitas mudanças e poucos resultados. Rev Saude Publica. 2011;45(5):949-63. http://dx.doi.org/10.1590/S0034-89102011005000069. PMid:21953026.

26. Malta DC, Mascarenhas MDM, Bernal RTI, da Silva MM, Pereira CA, Minayo MC, et al. Análise das ocorrências das lesões no trânsito e fatores relacionados segundo resultado da Pesquisa Nacional por amostra de Domicílio (PNAD) - Brasil, 2008. Cien Saude Colet. 2011;16(9):3679-87. http://dx.doi.org/10.1590/S141381232011001000005 . PMid:21987312.

27. Morais Neto OL, Montenegro MMS, Monteiro RA, et al. Mortalidade por acidentes de transporte terrestre no Brasil na última década: tendência e aglomerados de risco. Ciência Saúde Coletiva. 2012;17(9):22232236. http://dx.doi.org/10.1590/S1413-81232012000900002

28. Brasil. Lei no 9.503, de 23 de setembro de 1997. Institui o Código de Trânsito Brasileiro. Diário Oficial da União, Brasília, 23 de setembro de 1997.

29. World Health Organization. Global status report on alcohol and health [Internet]. 2011 [acesso 2019 mai 09]. Disponível em http://www.who.int/substance_abuse/publications/global_alcohol_report/ msbgsruprofiles.pdf 
30. World Health Organization. The epidemiology of road traffic accidentes. Geneva: WHO; 2014.

31. Malta DC, Bernal RTI, Mascarenhas MDM, da Silva MMA, Szwarcwald CL, de Morais Neto OL. Consumo de bebidas alcoólicas e direção de veículos nas capitais brasileiras e no Distrito Federal, segundo dois inquéritos nacionais de saúde. Rev Bras Epidemiol. 2015;18(Suppl. 2):214-23. http://dx.doi. org/10.1590/1980-5497201500060019. PMid:27008616.

32. Caixeta CR, Minamisava R, Oliveira LMAC, Brasil VV. Morbidade por acidente de transporte entre jovens de Goiânia, Goiás. Cien Saude Colet. 2010;15(4):2075-84. http://dx.doi.org/10.1590/S141381232010000400021. PMid:20694329.

33. Global Road Safety Partnership. Beber e Dirigir: manual de segurança viária para profissionais de trânsito e de saúde. Genebra: GRSP; 2007.

34. Damacena GN, Malta DC, Boccolini CS, Souza PR, Almeida WD, Ribeiro LS, et al. Consumo abusivo de álcool e envolvimento em acidentes de trânsito na população brasileira, 2013. Cien Saude Colet. 2016;21 (12):377786. http://dx.doi.org/10.1590/1413-812320152112.25692015. PMid:27925118.

35. Brasileiro IC, Moreira TMM. Prevalência de alterações funcionais corpóreas em crianças com paralisia cerebral, Fortaleza, Ceará, 2006. Acta Fisiátrica. 2008;15(1):37-41.

36. Oliveira LR, Macedo APFS, Buchalla CM, Scatena JHG. Evolution of disability in traffic accident victims in rehablitation, characterized by the International Classification of Functioning Disability and Health (ICF). Fisioterapia em Movimento. PUCPR. 2017;30:267-75.

37. Sabino GS, Coelho MC, Sampaio RF. Utilização da Classificação Internacional de Funcionalidade, Incapacidade e Saúde na avaliação fisioterapêutica de indivíduos com problemas musculoesqueléticos nos membros inferiores e região lombar. Acta Fisiátrica. 2008;15(1):24-30.

38. Lucena EMF, Morais JD, Batista HRL, et al. A funcionalidade de usuários acometidos por AVE em conformidade com a acessibilidade à reabilitação. Acta Fisiatr. 2011;18(3):112-8.

39. Sampaio RF, Luz MT. Funcionalidade e incapacidade humana: explorando o escopo da classificação internacional a Organização Mundial de Saúde. Cad Saude Publica. 2009;25(3):475-83. http://dx.doi. org/10.1590/S0102-311X2009000300002. PMid:19300836.

40. Diniz D, Medeiros M, Squinca F. Reflexões sobre a versão em Português da Classificação Internacional de Funcionalidade, Incapacidade e Saúde. Cad Saude Publica. 2007;23(10):2507-10. http://dx.doi.org/10.1590/ S0102-311X2007001000025. PMid:17891310.

41. Fontes AP, Fernandes AA, Botelho MA. Funcionalidade e incapacidade: aspectos conceptuais, estruturais e de aplicação da Classificação Internacional de Funcionalidade, Incapacidade e Saúde (CIF). Rev Port Saude Publica. 2010;28(2):171-8. http://dx.doi.org/10.1016/S0870-9025(10)70008-0.

42. Morettin M, Bevilacqua MC, Cardoso MRA. A aplicação da Classificação internacional de Funcionalidade, Incapacidade e Saúde (CIF) na Audiologia. Distúrb Comun. 2008;20(3):395-402.

43. Ruaro JÁ, Ruaro BR, Souza DE, et al. Panorama e perfil da utilização da CIF no Brasil - uma década de história. Braz J Phys Ther. 2012;16(6):454-62. http://dx.doi.org/10.1590/S1413-35552012005000063.

44. Athayde F, Mancuzo EV, Corrêa RA. Influência ambiental sobre a incapacidade física: uma revisão sistemática da literatura. Cien Saude Colet. 2017;22(11):3777-86. http://dx.doi.org/10.1590/1413812320172211.01992017. PMid:29211170. 